\title{
Cancer Survivorship Across the Healthcare Continuum
}

\author{
Adam Clark, PhD and Andy Miller, MHSE, CHES ${ }^{2}$ \\ 'Research and Policy, Lance Armstrong Foundation, Austin, TX, USA; ${ }^{2}$ Programs and Policy, Lance Armstrong Foundation, Austin, USA.
}

J Gen Intern Med 24(Suppl 2):503-4

DOI: $10.1007 / \mathrm{s} 11606-009-1089-3$

(c) Society of General Internal Medicine 2009

A $\mathrm{s}$ America struggles to enact meaningful health care reforms, there is great interest in and support for ensuring comprehensive, coordinated and patient-centered health care. But the challenges to delivering this type of health care are both great and systemic, ranging from regulation to reimbursement, and within the debate we risk the focus being taken off of the patient. Nowhere is this risk greater than for those diagnosed with cancer who rely upon a coordinated system to help them navigate multiple specialists and ensure the best, most timely care possible to save their lives. Fortunately, general internists are trained to treat patients as whole people, not as organ systems affected by disease, thus enabling them to approach this issue from a distinct perspective. As our understanding of quality cancer care increases and our focus expands to include cancer survivorship, we must ensure the patients' goals for their life after cancer are central to their care, not something to be dealt with as an afterthought once treatment is finished and the patient returns to the care of the general internist.

But in the current fragmented system, there do not seem to be sufficient incentives to prioritize the patient's needs, particularly those relevant to ensuring quality of life through the balance of the patient's life. General internists regularly see patients who are currently in treatment and either seeking relief from a whole host of side effects, including fatigue, or dealing with additional chronic illnesses. They are also in the unique position to help their patients navigate through the various players in the health care system and ensure they are being treated as a whole person, not simply as a tumor type.

Based on the number of people who will be diagnosed with cancer in their lifetime, if health system reform is to be effective, improving cancer survivorship must be a common goal throughout the healthcare continuum. It cannot be viewed solely as a separate or unrelated phase that occurs after treatment. This does not, however, mean that much of survivorship care is not provided after treatment. But our understanding of survivorship has evolved, and it should be viewed as an accompanying characteristic of each phase of the continuum, with the transition to post-treatment survivorship guided by thoughtful care planning that is relevant to the patient's unique experience. In this case, each physician participating in every step of the health care continuum should be asking, "Are my decisions and recommendations focused on improving survivorship for my patient?"
When we isolate survivorship as a stage occurring only after treatment for which someone else is responsible for addressing, we miss multiple opportunities to improve overall survivorship and center the care on the patient and their goals for treatment. How many times have we heard of patients being told of their potential infertility after treatment has been completed? Or patients discovering a clinical trial for their disease type in which they are interested in participating, only to learn they are ineligible due to having already begun chemotherapy. Imagine if every member of the health care team prioritized the patient's survivorship goals throughout the care continuum, communicating this with the patient and knowing that survivorship care planning will occur following treatment, we begin to see a more comprehensive and patient-centric model emerge. For the general internist, this means anticipating not only the side effects of treatment that they may be called upon to help manage, but also the long-term and late effects of cancer therapy that they will likely have to address once the patient completes treatment. Unfortunately, oncologists may be so focused on eliminating the patient's cancer, they may neglect to anticipate and prepare the patient for changes to their subsequent quality of life. It is here that the general internist can play the central role in caring for the whole patient, not simply their disease treatment.

We propose that improved survivorship is first dependent on prevention, screening, and early detection. And survivorship should inform diagnosis and treatment strategies, particularly as we move to molecular diagnostics, prognostics, and personalized medicine. The challenge is that the medical system from access to care, insurance coverage, physician reimbursement to follow-up care is a fractured process with many moving parts and players. But in order to achieve patientcentered cancer care, the next generation of our healthcare delivery system will need to support a comprehensive care model focused on survivorship.

Traditionally, the association between treatment and survivorship has been the easiest to understand, but to many it sounds counterintuitive to associate prevention directly with survivorship. But consider that prevention can take many forms, such as adapting clinical recommendations because of a family history or genetic predisposition to developing cancer, as in the case of carriers of the BRCA-1 mutations. Or in identifying precancerous lesions that may require continued monitoring and ongoing follow-up. And with the developing field of chemoprevention supporting new treatment systems in which pre-cancerous cells can be therapeutically managed and make the need for aggressive and toxic therapies unnecessary, we truly see a shift in focus to a quality-of-care delivery system based on making life better for the patient. General internists will be on the front line of addressing these issues with their patients, given that it will not be feasible for everyone at increased risk for cancer to be managed by an oncologist. 
Similar considerations should be taken into account with screening and early detection services. Discussions of survivorship should influence the conversation between the doctor and the patient and be initiated before a screening procedure is performed. This allows patients to be an active participant in managing their care and planning for their future. We know that one of the strongest predictors of whether an individual will get screened is if his or her physician recommends it. The lack of adherence with the screening recommendations has less to do with coverage or reimbursement than it has to do with prioritizing its importance to the individual patient and making sure he or she is comfortable with the screening procedures and potential outcomes.

For example, colorectal cancer has multiple proven screening options but nevertheless, adherence with recommended screening procedures is suboptimal. Each option has pros and cons associated with it, so the primary purpose should be to help the patient understand why screening is relevant for them and to use the method they are most comfortable with. It should be expected that patients will have different preferences in regards to screening methods, but the communication between the doctor and the patient should focus on how screening leads to improved survivorship.

Unfortunately, the conversation is not always easy. Unlike colorectal cancer screening which has a strong evidence base for improved survival and survivorship, prostate cancer screening can be more challenging. In this case, having a survivorship perspective is even more critical. Because prostate cancers are slow growing, it is not clear that screening improves overall survival. However, treatment of prostate cancer can have serious impact on a patient's quality of life and survivorship. The role of the physician in these cases is to help the patient understand the challenges of screening and all the options available. The choice of any medical intervention should be based on a patient's understanding all the options and potential long-term and late effects that research shows can significantly affect survivorship.

Finally, there is the overlap of survivorship considerations with treatment. As is the case with prostate cancer, the patient should understand all the options available, including participating in a clinical trial. Many times patients are only informed of the procedure their physician prefers to perform, not aware that there could be other options available. And unfortunately, increased costs of participating in a clinical trial can be more of a determining factor than patient choice. The treatment decision-making process is the time that survivorship care planning should truly begin and the general internist should be an active part of that planning, as the results of choices made now are those the general internist will have to address later. A patient should be aware of their options, potential complications or long-term and late effects, and work with their entire medical team to identify the approach that works best for them.

Clearly our healthcare system is still learning how to best translate the most up-to-date medical information into clinical care. And it is also becoming increasingly clear that the best care results from close coordination between the various specialists. But when that coordinated clinical care fails to make the patient's survivorship its primary goal, we lose the full benefits of truly comprehensive, patient-centered care. And based on their training to treat patients as a whole person, the general internist plays a critical role in coordinating this care, ensuring it becomes a reality for those who will live with a history of cancer. 